\title{
A review of studies concerning treatment adherence of patients with anxiety disorders
}

This article was published in the following Dove Press journal:

Patient Preference and Adherence

22 August 201I

Number of times this article has been viewed

Lívia Santana'

Leonardo F Fontenelle $e^{1-3}$

'Anxiety and Depression Research Program, Institute of Psychiatry, Universidade Federal do Rio de Janeiro, Brazil; ${ }^{2}$ Department of Psychiatry and Mental Health, Institute of Community Health, Universidade Federal Fluminense, Brazil; ${ }^{3} D^{\prime} O r$ Institute for Research and Education (IDOR), Rio de Janeiro, Brazil
Correspondence: Leonardo F Fontenelle Rua Visconde de Pirajá, 547 , Sala 719, Ipanema, Rio de Janeiro 22410-003, Brazil $\mathrm{Tel} / \mathrm{Fax}+552122394919$

Email fontenelle@gmail.com
Objective: This paper aimed at describing the most consistent correlates and/or predictors of nonadherence to treatment of patients with different anxiety disorders.

Method: The authors retrieved studies indexed in PubMed/MedLine, PsycINFO, and ISI Web of Knowledge using the following search terms: attrition OR dropout OR attrition rates OR patient dropouts OR treatment adherence AND anxiety disorders. Research was limited to articles published before January 2010.

Results: Sixteen studies were selected that investigated the impact of sociodemographic, clinical, or cognitive variables on adherence to treatment for anxiety disorders. While no consistent pattern of sociodemographic or clinical features associated with nonadherence emerged, all studies that investigated cognitive variables in panic disorder, social anxiety disorder, and obsessive-compulsive disorder found that expectations and opinions about treatment were related to adherence.

Conclusion: The findings of this study suggest that it is essential to consider anxiety disorder patients' beliefs about illness and treatment strategies to increase their compliance with the therapeutic plan.

Keywords: attrition, dropout, OCD, obsessive-compulsive disorder, social anxiety disorder

\section{Introduction}

Pathological anxiety and fear, ie, functioning impairing mood states associated with preparation for possible or imminent negative events, are the core features of the Diagnostic and Statistical Manual of Mental Disorders, Fourth Edition, Text Revision (DSM-IV-TR) anxiety disorders. Current conditions subsumed under its epithet include, among others, generalized anxiety disorder, obsessive-compulsive disorder (OCD), panic disorder, agoraphobia, specific phobias, social anxiety disorder (SAD) or "social phobia," posttraumatic stress disorder, and acute stress disorder. Epidemiological studies show that anxiety disorders are the most common class of mental disorders, affecting up to $28.8 \%$ of the general population at some point during their lives. ${ }^{1}$ People with anxiety disorders present significant functional and occupational impairments. In addition, they use public health services more often, thus leading to greater financial expenditures. ${ }^{1,2}$ These findings illustrate the importance of access to treatment and adherence to therapeutic strategies for patients with these conditions.

Although the dropout rate of patients with various psychiatric disorders for treatments in progress is approximately $50 \%,{ }^{3-5}$ the situation for anxiety disorders may be particularly problematic. For instance, it has been suggested that up to $85 \%$ of patients with social phobia who were initially interviewed do not attend follow-up 
treatment sessions. ${ }^{2}$ Although no clear information on the clinical, functional, and economic impact of treatment dropout anxiety disorders is available, the high level of attrition compromises the effectiveness of treatment. Identifying the risk factors for dropout in patients with anxiety disorders would allow clinicians to develop strategies that promote higher adherence to an established therapeutic plan (either pharmacological or psychosocial).

The objective of this review is to identify the sociodemographic, clinical, and cognitive variables that predict attrition/ dropout from different DSM-IV anxiety disorder treatments. Of note, studies including treatment adherence of patients with major depressive disorder with concomitant anxiety symptoms were excluded for not describing individuals with a primary anxiety disorder. The authors of this present review hypothesize that patients with lower socioeconomic levels, less education, more comorbidities, and negative beliefs or expectations regarding treatment will be more likely to drop out before completion compared with those without these characteristics.

\section{Methods}

The authors of this review identified studies that investigated predictors of attrition/dropout for anxiety disorder treatments through searches on PubMed/MedLine, PsycInfo, and ISI Web of Knowledge. The following search terms were used: attrition OR dropout OR attrition rates OR patient dropouts OR treatment adherence AND anxiety disorders. Research was restricted to articles published before January 2010. Additionally, the references of the selected studies were examined to find others related to the subject matter of interest.

Studies that investigated adherence to pharmacological, psychological, or both types of treatment in adults with a primary diagnosis of anxiety disorder were included. The studies that were included evaluated both the absence of treatment adherence after the initial interview but before the treatment had begun (ie, attrition) and the absence of treatment adherence after the treatment had begun (ie, "dropout"). Studies addressing attrition or dropout in randomized controlled trials, open studies, naturalistic follow-ups, and retrospective assessments were included. Excluded studies were those that (1) focused on appraising the attrition or dropout of treatment in patients with primary major depression associated with secondary anxiety, (2) described the index of attrition or dropout but did not evaluate its predictors, (3) investigated predictors of attrition or dropout in children or adolescents with anxiety disorders, and (4) included qualitative methods.

\section{Results}

A MedLine search resulted in 287 studies, of which 10 met the inclusion criteria. A PsycInfo search found 304 studies, of which two satisfied the inclusion criteria; however, these studies had already been selected in the MedLine search. Finally, an ISI Web of Knowledge search resulted in 318 articles, of which five met inclusion criteria, four of which had already been identified in the MedLine search. Another five relevant articles were found in the references of these studies that had not appeared in the database searches. Thus, a total of 16 articles were selected. A psychologist and a psychiatrist evaluated all of these articles jointly.

The data were organized into two subsections. The first part (Studies' designs) addressed the informative value of the reviewed studies, while the second one (Studies' results) described the results that can be inferred from these studies. The first subsection included tables listing studies that investigated treatment adherence in anxiety disorders in general (Table 1), in panic disorder (Table 2), in SAD (Table 3), and in OCD (Table 4), their sample sizes, the type of treatments they offered and/or were applied, the instruments that were employed, the attrition and dropout treatment indices, and the cognitive, clinical, and sociodemographic variables that predicted these features. The second subsection included one table (Table 5) describing the results associated with each potential predictor. This latter table describes the potential predictor, the number of studies investigating it, and the number of studies reporting a positive or negative association with adherence.

\section{Studies' designs}

A total of 16,766 patients with anxiety disorders were assessed with regard to treatment adherence, including 13,085 patients from a single retrospective study using a large managed care database. The impact of sociodemographic, clinical, and cognitive variables on adherence to treatment was evaluated in 14, 15, and seven studies, respectively. Seven papers assessed adherence to treatment in randomized controlled trials, six in naturalistic studies, three in open studies, and one in a retrospective study. One study combined data from an open and a controlled trial in a single analysis.

Most (12) studies assessed adherence to cognitive behavioral therapies, eight studies evaluated adherence to pharmacotherapy, and three investigated adherence to the combined treatment. Three papers included assessment of the three forms of treatment (pharmacotherapy, cognitive behavioral, or combined treatments). Treatment included serotonin reuptake inhibitors in four and individual cognitive behavioral treatment in five studies. Statistical analysis 


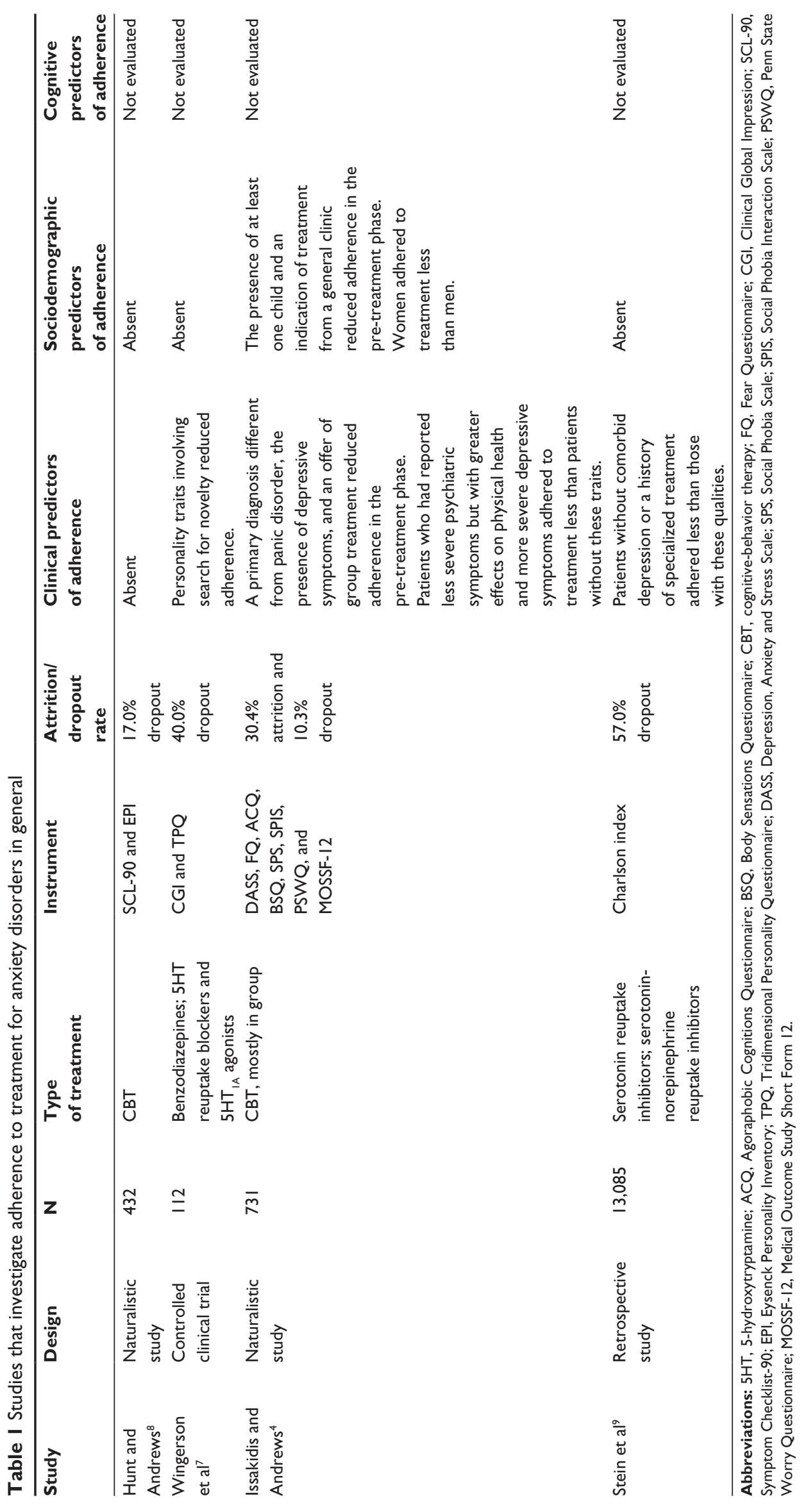




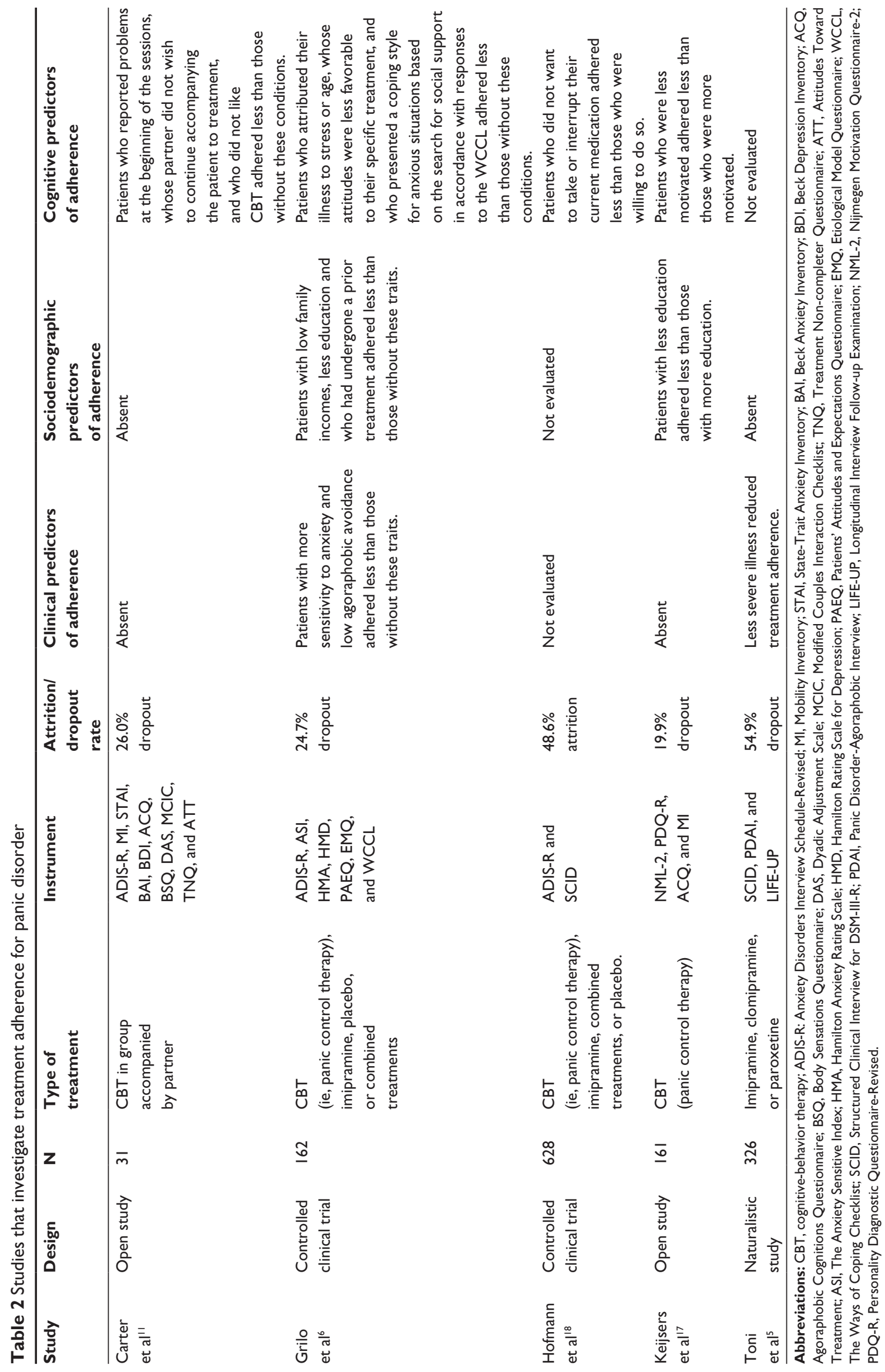




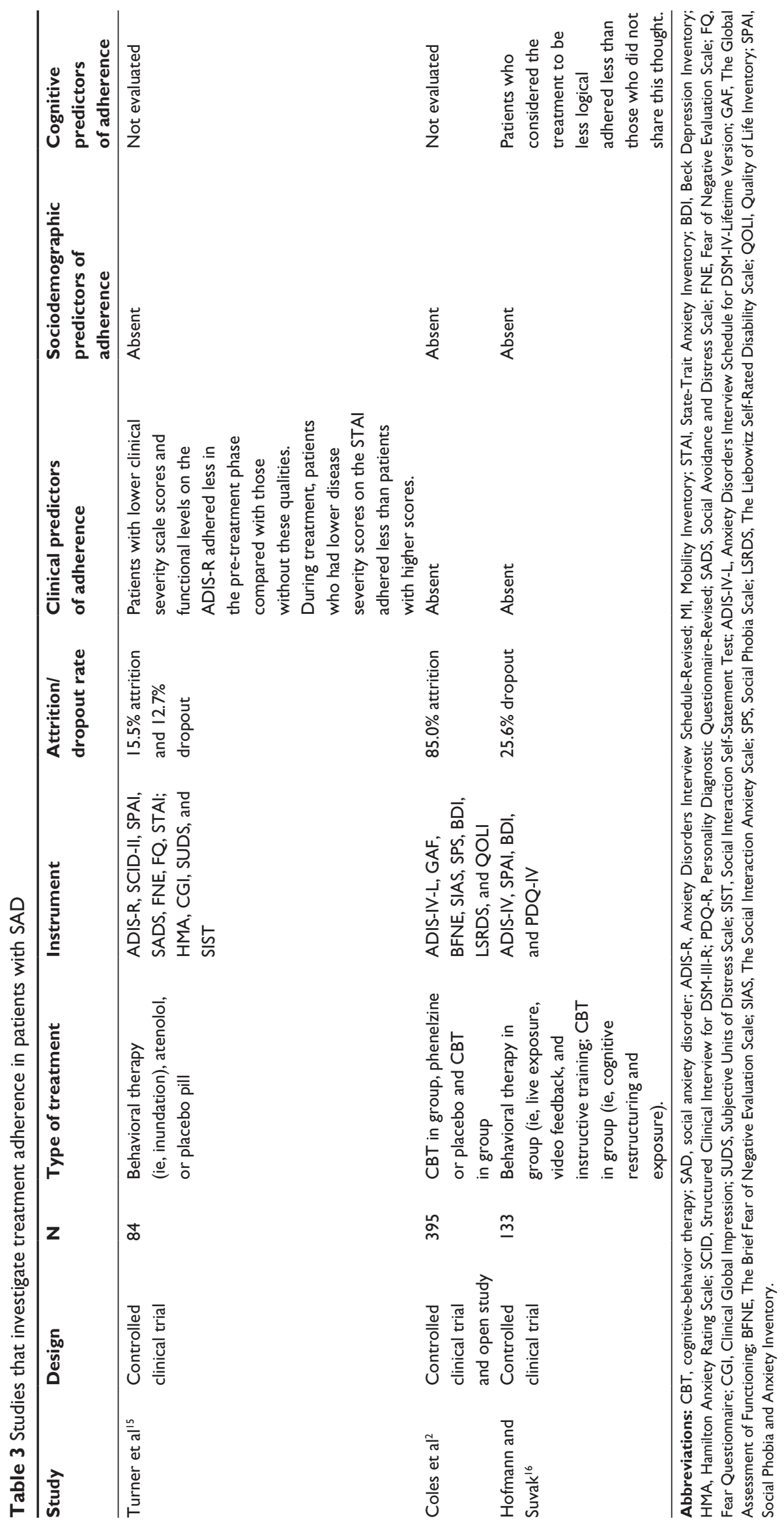




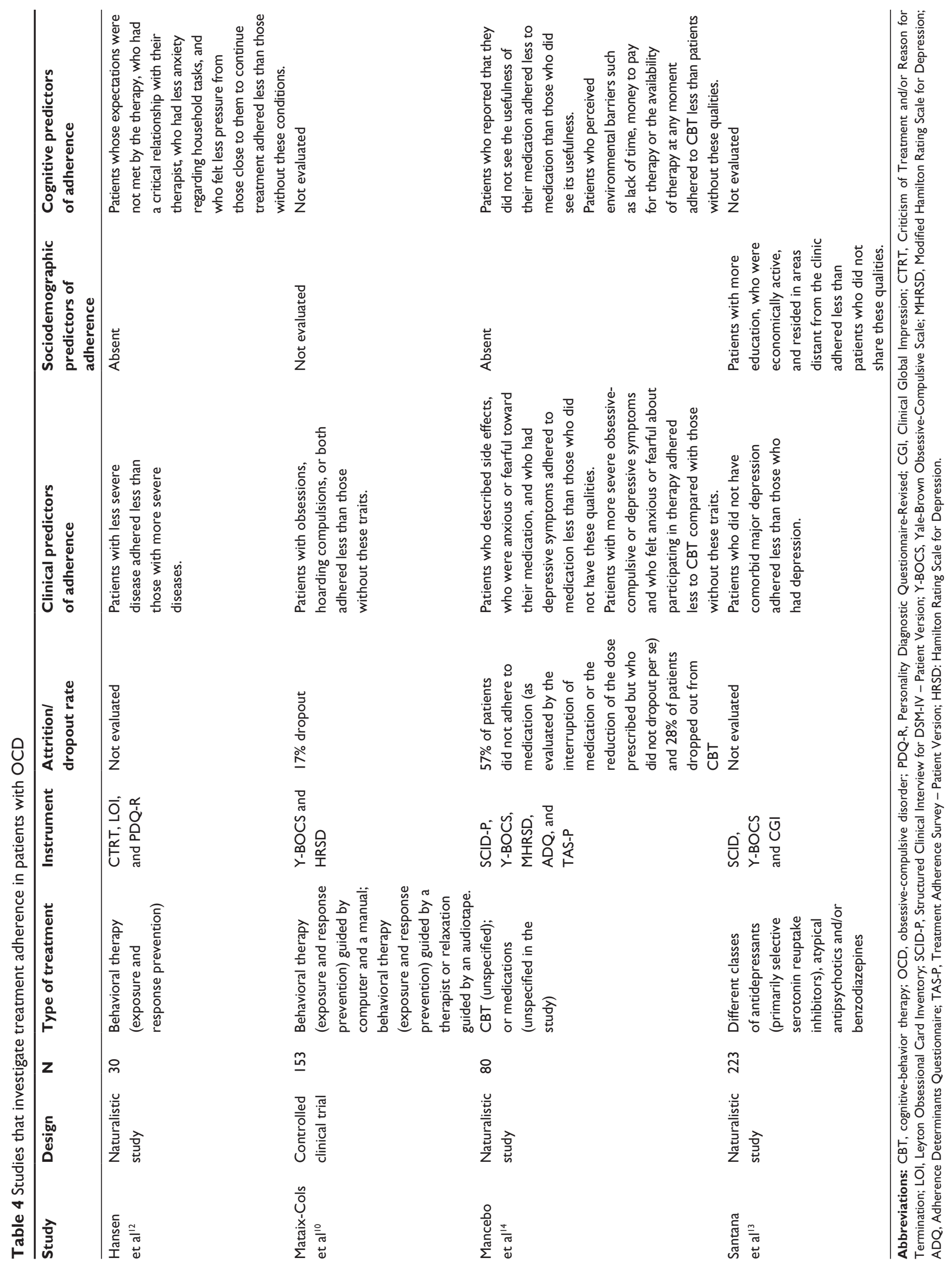


Table 5 Sociodemographic, clinical, and cognitive predictors of treatment adherence in anxiety disorders and the number of studies assessing them

\begin{tabular}{|c|c|c|c|c|}
\hline & $\begin{array}{l}\text { Potential } \\
\text { predictor }\end{array}$ & $\begin{array}{l}\text { Number } \\
\text { of studies }\end{array}$ & $\begin{array}{l}\text { Positive correlation } \\
\text { with dropout/attrition }\end{array}$ & $\begin{array}{l}\text { Negative correlation } \\
\text { with dropout/attrition }\end{array}$ \\
\hline \multicolumn{5}{|l|}{ Sociodemographic aspects } \\
\hline \multirow[t]{4}{*}{ Anxiety disorders in general } & Age & 14 & I & - \\
\hline & Female sex & 14 & I & - \\
\hline & Education level & 9 & I & 3 \\
\hline & Lower socioeconomic level & 6 & I & I \\
\hline \multicolumn{5}{|l|}{ Clinical aspects } \\
\hline \multirow[t]{3}{*}{ Anxiety disorders in general } & Severity of anxiety symptoms & 3 & - & I \\
\hline & Comorbidities & 3 & I & I \\
\hline & Personality factors & 2 & 1 & - \\
\hline \multirow[t]{3}{*}{ Panic disorder } & Severity of panic symptoms & 4 & I & I \\
\hline & Comorbidities & 2 & - & - \\
\hline & Personality factors & 3 & - & - \\
\hline \multirow[t]{3}{*}{ SAD } & Severity of SAD symptoms & 3 & - & I \\
\hline & Comorbidities & 3 & - & - \\
\hline & Personality factors & 2 & - & - \\
\hline \multirow[t]{3}{*}{ OCD } & Severity of OCD symptoms & 4 & 2 & I \\
\hline & Comorbidities & 2 & I & I \\
\hline & Personality factors & 1 & - & - \\
\hline \multicolumn{5}{|l|}{ Cognitive aspects } \\
\hline Panic disorder & & 4 & 4 & - \\
\hline SAD & & 1 & 1 & - \\
\hline OCD & & 2 & 2 & - \\
\hline
\end{tabular}

Abbreviations: $O C D$, obsessive-compulsive disorder; SAD, social anxiety disorder.

also varied greatly, but most studies used chi-square tests, regression analysis, and correlations to find the predictors (or correlates) of adherence.

\section{Studies' results}

A summary of findings, describing different classes of sociodemographic, clinical, and cognitive predictors of treatment adherence, is depicted in Table 5, along with the number of studies assessing each dimension and the number of studies showing a positive and negative association between each predictor and treatment adherence. Of note, for the sake of clarity, the data on sociodemographic aspects of different anxiety disorders and the severity of key anxiety symptoms, comorbidities, and personality factors have been collapsed into single variables for each anxiety disorder.

\section{Sociodemographic variables Age}

Fourteen studies attempted to evaluate the impact of participants' age on treatment adherence..$^{2,4-16}$ Coles et $\mathrm{al}^{2}$ investigated the pre-treatment phase of a trial of group cognitive-behavior therapy (CBT), phenelzine, and placebo for SAD. They found that these patients displayed a high pretreatment attrition rate $(85 \%)$ and that older patients turned down treatment more often than younger ones.
Sex

The same studies assessed the impact of participants' sex on treatment adherence. ${ }^{2,4-16}$

Among these studies, Issakidis and Andrews ${ }^{4}$ studied patients with various anxiety disorders and found that women dropped out of CBT more frequently than men.

\section{Education level}

Nine studies investigated the influence of education level on treatment adherence in patients with anxiety disorders. ${ }^{2,4,6,11,13-17}$ Of these studies, four found significant results. ${ }^{2,6,13,17}$ In a univariate analysis, Grilo et $\mathrm{al}^{6}$ found that patients with panic disorder who dropped out of a treatment trial comparing CBT, imipramine, and placebo had less education than those who completed treatment. However, a multivariate regression that controlled for other variables (eg, sociodemographic characteristics, severity of panic disorder, psychiatric comorbidity, attitude toward treatment, coping style, and personality style) found that educational level did not significantly contribute to dropouts, unlike family income and participant age.

In the Coles et $\mathrm{al}^{2} \mathrm{SAD}$ study, treatment-seeking people with less education, who were non-Caucasian and either unemployed or employed fulltime, were significantly more likely to schedule, but not attend, an initial interview. 
Furthermore, Coles et $\mathrm{al}^{2}$ conducted a logistic regression to determine the extent to which demographic variables determined interview attendance. A model that included race, age, and level of education explained $70.7 \%$ of the variance of treatment adherence.

Keijsers et $\mathrm{al}^{17}$ studied 161 patients with panic disorder and also found that educational level predicted treatment adherence to CBT (panic control therapy). They found that less education was associated with dropping out of treatment. Although educational level did not emerge as a predictor in a univariate analysis, a regression model that included motivation found that educational level was significantly associated with dropping out of treatment.

Unlike the studies above, Santana et $\mathrm{al}^{13}$ conducted a naturalistic follow-up study on 223 patients with OCD at a university clinic for anxiety disorders that offered free pharmacological treatment. In that study, the follow-up time of the patients (up to 10 years) was considered to be a measure of treatment adherence. Through a logistic regression, the authors of that study found that patients with less education remained in treatment longer.

\section{Socioeconomic level}

Seven studies investigated socioeconomic level, including family income and employment status. , $, 4,6,7,13,16$ Two studies found significant differences between adherent and nonadherent patients in terms of socioeconomic levels. ${ }^{6,13}$ As previously described, Grilo et $\mathrm{al}^{6}$ found that panic disorder patients who dropped out of a treatment trial comparing CBT, imipramine, and placebo presented a significantly lower income than patients who continued treatment. These findings were detected with regression models. On the other hand, Santana et $\mathrm{al}^{13}$ used a linear regression model to find that unemployed OCD patients remained in treatment longer at a public service compared with those with jobs.

\section{Other sociodemographic variables}

Santana et $\mathrm{al}^{13}$ examined 223 patients with OCD and found a relationship between the patients' places of residence and treatment adherence. Patients who lived in the city where the clinic was located remained in treatment longer than those who lived in a different city. Issakidis and Andrews ${ }^{4}$ used regression models to observe that patients with different anxiety disorders who had at least one child and who were treated at a general clinic rather than by a specialist in a mental health clinic were more likely to turn down the pre-treatment phase of CBT.
Similarly, in a study on adherence to pharmacological treatments in 13,085 patients with anxiety disorders, Stein et $\mathrm{al}^{9}$ observed that patients who were treated by a mental health specialist adhered to treatment more than those who were not seen by a specialist. Finally, Grilo et $\mathrm{al}^{6}$ found that panic disorder patients with a history of previous and brief treatments, defined by a Likert scale varying from 1 (no previous treatment) to 4 (more than a year of treatment), were more susceptible to dropping out from a controlled treatment trial with CBT, imipramine, and placebo compared with patients without this history.

\section{Clinical variables}

\section{Anxiety disorders in general}

Hunt and Andrews ${ }^{8}$ investigated 1045 patients who sought CBT for anxiety disorders from 1986 to 1988. Of these patients, 546 met the diagnostic criteria of panic disorder, agoraphobia, SAD, and generalized anxiety disorder. Of these patients, 432 patients accepted treatment. The clinical variables investigated were diagnosis, the severity of psychiatric symptom scale score (Symptoms Checklist-90 or SCL-90), the locus of behavioral control scale score and the Eysenck Personality Inventory (EPI) neuroticism subscale score. There were no significant differences found between the group who completed treatment $(n=357)$ and those who dropped out of treatment $(n=75)$.

Wingerson et $\mathrm{al}^{7}$ hypothesized that personality factors might contribute to dropping out of treatment. They investigated 112 patients with anxiety disorders treated with pharmacotherapy (including 5-hydroxytryptamine (5HT) reuptake blockers, benzodiazepines, and $5 \mathrm{HT}_{1 \mathrm{~A}}$ agonists). For panic disorder and generalized anxiety disorder patients combined, early dropouts scored higher on total novelty seeking of the Tridimensional Personality Questionnaire, as well as on the novelty-seeking traits of both disorderliness/ dislike of regimentation and impulsiveness. Patients who dropped out of treatment (40\%) did not differ from those who remained with regard to history of depression, alcohol or drug abuse, psychiatric hospitalization, attempted suicide, or symptom severity.

Issakidis and Andrews ${ }^{4}$ investigated 731 patients who sought and received treatment in a clinic specialized in anxiety disorders. To analyze the data, they defined two dependent variables: attrition in pre-treatment (either refusal of treatment or nonappearance) and dropping out of treatment once it has started. They analyzed primary psychiatric diagnosis, severity of symptoms, psychiatric comorbidities, and degree of incapacity. Attrition at pre-treatment (30.4\%) 
was associated with primary diagnosis because patients with depression or another psychiatric disorder were more likely to turn down treatment compared with those with panic disorder. In addition, the presence of more severe depressive symptoms at the initial interview and selection for group (rather than individual) treatment also significantly predicted attrition. In total, $10.3 \%$ of patients dropped out of treatment, and as in the pre-treatment phase, patients with comorbid depression or depressive symptoms dropped out of treatment more often than those without these symptoms. Dropouts also reported less severe symptoms before treatment but a greater impairment of physical health.

Stein et $\mathrm{al}^{9}$ examined patients with various anxiety disorders who were offered pharmacological treatments and found divergent results from those of Issakidis and Andrews. ${ }^{4}$ According to Stein et al, ${ }^{9}$ patients with comorbid depression adhered to antidepressants (5HT and serotoninnoradrenaline reuptake blockers) more than those without such a comorbidity. They argued that a possible reason for the lower rates of adherence in the nondepressed group was the lower likelihood of mental health specialty care within this population. In their analysis, ${ }^{9}$ significantly fewer patients with anxiety alone, compared with patients with comorbid depression, received mental health specialty care during the year after treatment initiation.

In summary, studies that investigated anxiety disorders in general differed from each other with regard to their methodologies and results. The prevalence of dropouts varied from $10.3 \%$ to $57.0 \%$. Only one study investigated attrition during pre-treatment. ${ }^{4}$ All of the studies investigated the impact of comorbidities and the severity of symptoms ${ }^{4,7-9}$ in adherence. However, only two studies ${ }^{4,9}$ found significant differences between adherent and nonadherent patients, with divergent results. Specifically, one study found that patients with comorbid depression were more likely to turn down pre-treatment and drop out from treatment ${ }^{4}$ compared with patients without comorbidities, whereas another study found that patients with comorbid depression were more likely to adhere to treatment. ${ }^{9}$

\section{Panic disorder}

Carter et al $^{11}$ investigated 31 patients with panic disorder and agoraphobia who were offered group CBT. The patients' partners accompanied them to each session. The clinical variables investigated were the number of situations avoided and the severity of their panic disorder; however, no significant differences were found through a multivariate analysis.

Grilo et $\mathrm{al}^{6}$ investigated 162 patients diagnosed with panic disorder, with or without agoraphobia, who were offered
CBT, medication, or both. They investigated six domains of variables: sociodemographic characteristics, severity of panic disorder, psychiatric comorbidities, attitude toward treatment, coping style, and personality style. After conducting multivariate regressions, the authors of that study verified that patients with higher Anxiety Sensitivity Index (ASI) scores and low agoraphobic avoidance were more likely to discontinue treatment.

As in the study above, Keijsers et $\mathrm{al}^{17}$ investigated 161 patients with panic disorder who were offered CBT. The clinical variables investigated were the severity of symptoms and dysfunctional personality traits. The severity of symptoms was evaluated through three characteristics: catastrophic agoraphobic cognition, agoraphobic avoidance behavior, and the frequency of panic attacks via the Agoraphobic Cognitions Questionnaire and the Mobility Inventory. Furthermore, these authors of that study evaluated psychopathic personality traits using the Personality Diagnostic Questionnaire-Revised to investigate whether patients with personality disorders or with higher scores on this scale were more likely to discontinue treatment. They found no significant differences between dropouts (19.9\%) and those who completed treatment.

Toni et $\mathrm{al}^{5}$ investigated 326 patients diagnosed with panic disorder who were treated with antidepressants and followed up for 3 years. During this period, 179 (54.9\%) patients dropped out of treatment. The only statistically significant difference between dropouts and those who completed treatment was a lower severity of panic disorder among the first group.

To summarize, five studies investigated treatment adherence in patients with panic disorder. ${ }^{5,6,11,17,18}$ Among this sample, four studies ${ }^{5,6,11,17}$ investigated clinical variables related to dropouts in patients with panic disorder and three studies included CBT. ${ }^{6,11,17}$ All of the studies evaluated the severity of panic disorder, but only two studies found significant findings, and these had divergent results. In one study, ${ }^{6}$ patients with a more severe disorder withdrew from treatment more often than patients with a less severe disorder, whereas the reverse was true in the other study. ${ }^{5}$ None of these four studies $^{5,6,11,17}$ evaluated attrition in pre-treatment.

\section{SAD}

Turner et $\mathrm{al}^{15}$ investigated 84 patients with SAD who met the criteria for generalized or circumscribed forms. These patients were randomly divided into three groups: (1) exposure, (2) atenolol, and (3) placebo pill. Thirteen $(15.5 \%)$ patients selected for this study declined to participate 
in the treatment protocol. These patients presented lower severity scores on the Anxiety Diagnostic Interview Schedule (ADIS)-Reviewed scale. Among those who participated in this study, nine $(12.7 \%)$ patients dropped out of treatment over the course of the 12-week program and differed from those who completed treatment in terms of a lower disorder severity as measured by the Fear Questionnaire Symptom Severity Scale and the Spielberger State-Trait Anxiety Inventory self-reports.

Coles et $\mathrm{al}^{2}$ analyzed the treatment course for patients with SAD from the initial telephone contact to the beginning of treatment. A total of 395 people made an initial telephone call; of these people, only 60 began treatment. Thus, $85 \%$ of the patients who made initial contact with the clinic did not begin treatment. Of the 395 patients who contacted the clinic, 131 completed an initial interview. Of these patients, 126 people were diagnosed with SAD, and 60 patients accepted and began treatment. No significant differences were found between the group who began treatment $(n=60)$ and those who did not $(n=66)$ with regard to the following clinical variables: severity of symptoms, depressive symptoms, quality of life (as measured by the Quality of Life Inventory), and functional impairment (as measured by the Liebowitz Self-Rated Disability Scale).

Hofmann and Suvak ${ }^{16}$ also investigated 133 patients with SAD who sought group behavioral therapy or group CBT in a center for anxiety at Boston University. Of the 133 patients, 34 (25.6\%) dropped out of treatment. The authors of that study investigated the following clinical variables: DSM-IV Axis-I diagnoses, anxiety and depression scores (ie, the Social Phobia and Anxiety Inventory and the Beck Depression Inventory, respectively), personality disorders, and the index of disease severity stemming from social phobia (as measured by the ADIS for DSM-IV [ADIS-IV]). They compared the clinical and sociodemographic variables of the group that completed treatment with those of the group who dropped out and did not find significant differences.

In summary, all of the studies reviewed here investigated the influence of clinical variables on the adherence to treatment for SAD. ${ }^{2,15,16}$ Two studies ${ }^{2,15}$ analyzed attrition from treatment and obtained different results. Turner et al ${ }^{15}$ found that $15 \%$ of patients withdrew from study participation. Coles et $\mathrm{al}^{2}$ found that $85 \%$ of people who sought clinical help withdrew before treatment. This between-study heterogeneity may be partially explained by the disparate approaches that the studies used to conduct their investigations. Coles et $\mathrm{al}^{2}$ began their investigation with the first phone call for treatment, whereas Turner et al ${ }^{15}$ investigated attrition in patients who already had a confirmed diagnosis and passed through the initial stages of treatment but who ultimately declined to participate. Two studies ${ }^{15,16}$ investigated the patients who began treatment and dropped out; however, only one found that patients with less severe disease symptoms were more likely to discontinue treatment. ${ }^{15}$

\section{OCD}

Hansen et $\mathrm{al}^{12}$ investigated nonadherence to therapy in patients with OCD who had undergone CBT. They compared groups of 15 dropouts and 15 patients who successfully completed a treatment that consisted of 10 sessions of exposure and response prevention. Patients who abandoned treatment without informing their therapist during the study period were considered to be nonadherent. The researchers analyzed the severity of OCD as measured by the Leyton Obsessional Inventory (LOI) and personality disorders determined using the Personality Diagnostic Questionnaire-Revised. Patients who discontinued treatment had lower LOI disease-severity scores.

Mataix-Cols et $\mathrm{al}^{10}$ investigated whether different symptom dimensions in patients with OCD were associated with treatment adherence and behavioral therapy response. They investigated patients who participated in a controlled clinical trial of behavioral therapy either by computer or guided by a clinician. They randomly selected 218 patients with OCD to receive 10 sessions of treatment for (1) exposure and response prevention guided by computer and a manual, (2) exposure and response prevention guided by a behavioral therapist, and (3) relaxation guided by audiotape. After a 3-month follow-up, the patients in the first two groups (exposure by computer or therapist) similarly improved, and both groups improved more than the relaxation control group. Of the patients who received at least one visit for post-evaluation, 153 completed the Yale-Brown Obsessive-Compulsive Scale Checklist (Y-BOCS). The severity of obsessive-compulsive symptoms at the beginning of treatment predicted response to therapy, and serious symptoms at the outset of therapy continued to be more critical at the conclusion of treatment. Patients with high scores on the sexual/religious dimension of the Y-BOCS had poorer responses to exposure and response prevention treatment. Regression analyses revealed that high scores on the hoarding dimension predicted dropouts.

Mancebo et $\mathrm{al}^{14}$ validated an instrument (ie, the Treatment Adherence Survey - patient version) that investigates adherence to pharmacological and psychological treatments in patients with OCD. This instrument is composed of two parts. The first section investigates adherence to CBT, and the second section investigates adherence to pharmacotherapy. 
Mancebo et $\mathrm{al}^{14}$ selected 80 patients who participated in the Brown Longitudinal OCD Study. Of these patients, 28\% did not adhere to CBT and $57 \%$ did not adhere to medication. Those who did not adhere to CBT replied that they felt too anxious or fearful to participate in therapy (55\%). These same patients also had more severe obsessive-compulsive and depressive symptoms as evaluated by the Y-BOCS and the Modified Hamilton Rating Scale for Depression, respectively. Patients who did not adhere to medication more frequently reported dissatisfaction with side effects (77\%) and anxiety or fear with respect to taking medication (41\%). These patients also had more severe depressive symptoms than those who adhered to treatment.

Santana et al $^{13}$ also investigated patients with OCD and, similarly to Hansen et al, ${ }^{12}$ found results that diverged from those of Mancebo et al. ${ }^{14}$ In this naturalistic study, the only clinical variable that predicted adherence was comorbid major depression. However, patients who presented with this comorbidity were more likely to adhere to treatment.

In summary, all the studies that investigated adherence to OCD treatment evaluated the impact of the disease's severity. ${ }^{10,12-14}$ The results were divergent: Hansen et al ${ }^{12}$ found that patients with less severe obsessive-compulsive symptoms more frequently discontinued treatment, whereas Mancebo et $\mathrm{al}^{14}$ found that patients with more severe symptoms more frequently dropped out of treatment. Mataix-Cols et $\mathrm{al}^{10}$ found that the severity of OCD predicted response to treatment but not adherence. One study ${ }^{14}$ observed that patients with comorbid depression and more severe depressive symptoms adhered to treatment less often than those without these symptoms, while another one ${ }^{13}$ described that comorbid depression increased adherence.

\section{Cognitive variables}

\section{Panic disorder}

Carter et $\mathrm{al}^{11}$ asked patients with panic disorder who dropped out CBT in group to complete a self-report questionnaire. These patients claimed not to have been satisfied with their treatment. In addition, they stated that their partner, who functioned as their co-therapist and accompanied them to all the sessions, did not want them to continue treatment. Finally, they declared having "difficulties with the therapy sessions." This last response appeared on a self-report questionnaire (Treatment Non-completer Questionnaire) that lists 18 common reasons that influence interruptions of treatment; however, the authors of the study did not provide explanations of these "difficulties."
Grilo et $a l^{6}$ found significant differences in the attitudes of dropout panic disorder patients regarding their treatment and the reasons they provided with respect to the etiology of their panic attacks. Patients who (1) attributed their panic attacks to life stressors, (2) had less favorable attitudes toward their treatment (group versus individual treatment), and (3) used a coping style based on social support were more likely to discontinue treatment.

Hofmann and Suvak ${ }^{16}$ investigated attrition in patients with panic disorder who sought, or were referred to, two clinics during the pre-treatment phase. One of the clinics offered a pharmacological treatment, whereas the other offered a psychological treatment. After an initial interview, during which patients were assessed for a formal DSM-IV diagnosis of panic disorder, they participated in a study that offered five randomly distributed treatment modalities: (1) imipramine, (2) panic control (ie, psychological treatment), (3) placebo, (4) imipramine and panic control, and (5) placebo and panic control. The patients were informed that they had a $92 \%$ chance of receiving an active treatment. Of 628 pre-selected patients, 115 were ultimately excluded from the study due to diagnosis changes, medical problems, or other reasons. Of those eligible for treatment, 305 patients $(48.6 \%)$ refused to participate. The principal reasons given by the patients who turned down treatment were that they were not willing to take medication $(33.8 \%)$ or that they were not willing to interrupt their usual medication schedule (24.9\%). The latter reason occurred due to a study regulation in which participants were required to interrupt the use of all current psychotropic medications.

Keijsers et $\mathrm{al}^{17}$ also found that patients with panic disorder who were less motivated for treatment were significantly more likely to discontinue treatment. This result appeared in both a univariate analysis and a regression model. In addition, the authors of that study contacted 25 of the 32 dropouts; the most common reasons these former patients provided for dropping out included dissatisfaction with CBT and its protocol and their improvement to that point, as well as a lack of motivation.

\section{SAD}

Hofmann and Suvak ${ }^{16}$ found that SAD patients who discontinued behavioral or group CBT treatment found therapy to be less logical than those who completed treatment. A selfreport questionnaire administered after the first session also investigated the attitude of the patients toward their treatment. Hofmann and Suvak ${ }^{16}$ tried to contact patients who discontinued treatment, but only $50 \%$ responded. Despite a positive 
correlation between "lack of logic" and the dropout rate, the patients reported that they discontinued treatment because of conflicts with work, feelings that the group environment was overwhelming or that the treatment was ineffective, moving to a different city, and personal reasons.

\section{OCD}

Hansen et $\mathrm{al}^{12}$ studied patients with OCD after a psychological treatment and found similar reasons for dropout using a structured questionnaire. Dropouts stated that the therapy did not correspond to their expectations and had criticisms concerning their therapist. Furthermore, these former patients felt less "pressure" from someone close to them to continue therapy compared with those who completed treatment. These results highlight the importance of knowing and agreeing to the proposed treatment, patients' relationships with their therapist, and their attitudes toward treatment.

Mancebo et $\mathrm{al}^{14}$ found that 80 patients with OCD reported perceived environmental barriers between CBT and their ability to complete treatment. Although there were no explanations of these "environmental barriers," the fact that the patients perceived them as a reason for not adhering to treatment indicates the presence of beliefs with regard to treatment access which must be better investigated. With regard to medication adherence, these patients also questioned the usefulness of their medication.

\section{Discussion}

A total of 16 studies were selected that investigated the impact of sociodemographic or clinical variables on adherence to treatment for anxiety disorders. The greatest majority of the available studies were unable to find sociodemographic differences between adherent and nonadherent patients with anxiety disorders. Only one naturalistic study reported that women with anxiety disorders discontinued treatment more frequently than men. ${ }^{4}$ Further, in just one study, older, non-Caucasian and unemployed anxiety disorder patients displayed treatment attrition more frequently than those who did not share these features. ${ }^{2}$ However, in a naturalistic study, unemployed OCD patients were more likely to adhere to treatment. ${ }^{13}$ Therefore, it seems that no consistent conclusion regarding the effect of gender, age, race, and employment on adherence of patients with anxiety disorders to treatment can be reached at this moment.

Nine studies ${ }^{2,4,6,11,13-17}$ investigated the influence of education level on treatment adherence of patients with anxiety disorders, but only four ${ }^{2,4,13,17}$ reported significant findings. Specifically, three studies found that patients with less education were more likely to turn down treatment, ${ }^{2,4,17}$ whereas one study found the opposite result. ${ }^{13}$ The authors of this present review believe that the interpretation of these contradictory findings should take into account the peculiarities of each mental health service in question. For instance, while greater levels of education could foster adherence to a private and/or wealthy clinic, it could also lead to greater dropout rates in a public service dedicated to lower income individuals.

Further, six studies $2,4,6,13,16$ investigated the impact of socioeconomic levels on treatment adherence in anxiety disorders. Of note, only one study on patients with panic disorder found an association between lower socioeconomic level and higher likelihoods of dropout, ${ }^{6}$ while a naturalistic study of OCD found a paradoxical association between higher socioeconomic levels and greater adherence to treatment. ${ }^{13}$ In the latter study, Santana et al ${ }^{13}$ tracked patients for 10 years via a free university service. Thus, patients with more education and higher socioeconomic levels might have sought treatment in private services to avoid some of the inconveniences of public services such as lines and hours spent waiting for the appointment.

In summary, although there is some evidence that both lower educational levels and socioeconomic status can adversely affect adherence in anxiety disorders, studies showing no relationship outnumber those that do. Also, the relationship between adherence to treatment and educational levels and socioeconomic status need to be examined in the light of the characteristics of the service being provided.

Fifteen studies evaluated the impact of clinical variables on dropping out of treatment. ${ }^{2,4-7,8-17}$ Despite the fact that many studies of depression show that comorbidity predicts adherence to treatment, the present review revealed that only four studies found a relationship between comorbidity and treatment adherence. Furthermore, the results were divergent. Stein et $\mathrm{al}^{9}$ and Santana et $\mathrm{al}^{13}$ found that patients with comorbid depression adhere to treatment more often than those without this comorbidity. In contrast, Issakidis et $\mathrm{al}^{4}$ and Mancebo et $\mathrm{al}^{14}$ found that patients with comorbid depression adhere less to treatment. Thus, our hypothesis that comorbidity would predict adherence was not confirmed based on studies with depressed patients.

The five studies that investigated cognitive variables found similar results and emphasized the importance of treatment choice and favorable attitudes toward treatment. $6,11,14,16,18$ Cognitive variables may be an important intervention factor because they are more modifiable than clinical and sociodemographic variables. The results of this review suggest 
that treatment programs need to consider these expectations and include structured interventions to motivate patients to participate in treatment even before it begins.

\section{Conclusion}

Few studies have researched correlates or predictors of attrition and/or dropout in patients with anxiety disorders. The heterogeneity of the findings described in this review partially reflects the different methodologies used to identify the factors involved in the treatment adherence of patients with anxiety disorders. For example, many studies analyzed the effects of pharmacological and psychological treatments separately, whereas other studies investigated combined treatments. Also, given that researchers conceptualize "adherence" in different ways, a consensus definition is necessary. For example, although some researchers consider dropping out to be the cessation of treatment before its official conclusion, others consider dropouts to be people who continue to attend treatment but do not appear at all of the sessions or who take medication less frequently or in smaller doses than those prescribed.

Despite this review's negative findings regarding the impact of sociodemographic and clinical features of patients with anxiety disorders in treatment adherence, the studies are rather consistent in describing a high rate of nonadherence among patients with anxiety disorders. ${ }^{9}$ Thus, the authors of this present review emphasize the importance of more research on this topic in order to develop strategies that help patients conclude their treatments. Furthermore, they emphasize this review's consistent results on cognitive variables, which indicate that expectations and beliefs about the disease and its treatment are important features to be considered in the management of patients with anxiety disorders.

\section{Disclosure}

The authors report no conflicts of interest in this work.

\section{References}

1. Kessler RC, Berglund P, Demler O, Jin R, Merikangas KR, Walters EE. Lifetime prevalence and age-of-onset distributions of DSM-IV disorders in the National Comorbidity Survey Replication. Arch Gen Psychiatry. 2005;62(6):593-602.

Patient Preference and Adherence

\section{Publish your work in this journal}

Patient Preference and Adherence is an international, peer-reviewed, open access journal focusing on the growing importance of patient preference and adherence throughout the therapeutic continuum. Patient satisfaction, acceptability, quality of life, compliance, persistence and their role in developing new therapeutic modalities and compounds to
2. Coles ME, Turk CL, Jindra L, Heimberg RG. The path from initial inquiry to initiation of treatment for social anxiety disorder in an anxiety disorders specialty clinic. J Anxiety Disord. 2004;18(3):371-383.

3. Young BJ, Beidel DC, Turner SM, Ammerman RT, McGraw K, Coaston SC. Pretreatment attrition and childhood social phobia: parental concerns about medication. J Anxiety Disord. 2006;20(8): 1133-1147.

4. Issakidis C, Andrews G. Pretreatment attrition and dropout in an outpatient clinic for anxiety disorders. Acta Psychiatr Scand. 2004; 109(6):426-433

5. Toni C, Perugi G, Frare F, Mata B, Akiskal HS. Spontaneous treatment discontinuation in panic disorder patients treated with antidepressants. Acta Psychiatr Scand. 2004;110(2):130-137.

6. Grilo CM, Money R, Barlow DH, et al. Pretreatment patient factors predicting attrition from a multicenter randomized controlled treatment study for panic disorder. Compr Psychiatry. 1998;39(6):323-332.

7. Wingerson D, Sullivan M, Dager S, Flick S, Dunner D, Roy-Byrne P. Personality traits and early discontinuation from clinical trials in anxious patients. J Clin Psychopharmacol. 1993;13(3):194-197.

8. Hunt C, Andrews G. Drop-out rate as a performance indicator in psychotherapy. Acta Psychiatr Scand. 1992;85(4):275-278.

9. Stein MB, Cantrell CR, Sokol MC, Eaddy MT, Shah MB. Antidepressant adherence and medical resource use among managed care patients with anxiety disorders. Psychiatr Serv. 2006;57(5):673-680.

10. Mataix-Cols D, Marks IM, Greist JH, Kobak KA, Baer L. Obsessivecompulsive symptom dimensions as predictors of compliance with and response to behaviour therapy: results from a controlled trial. Psychother Psychosom. 2002;71(5):255-262.

11. Carter MM, Turovsky J, Sbrocco T, Meadows EA, Barlow DH. Patient dropout from a couples group treatment for panic disorder with agoraphobia. Prof Psychol Res Pract. 1995;26(6):626-628.

12. Hansen AM, Hoogduin CA, Schaap C, de Haan E. Do drop-outs differ from successfully treated obsessive-compulsives? Behav Res Ther. 1992;30(5):547-550.

13. Santana L, Versiani M, Mendlowicz MV, Fontenelle LF. Predictors of adherence among patients with obsessive-compulsive disorder undergoing naturalistic pharmacotherapy. J Clin Psychopharmacol. 2010;30(1):86-88.

14. Mancebo MC, Pinto A, Rasmussen SA, Eisen JL. Development of the Treatment Adherence Survey-patient version (TAS-P) for OCD. J Anxiety Disord. 2008;22(1):32-43.

15. Turner SM, Beidel DC, Wolff PL, Spaulding S, Jacob RG. Clinical features affecting treatment outcome in social phobia. Behav Res Ther. 1996;34(10):795-804.

16. Hofmann SG, Suvak M. Treatment attrition during group therapy for social phobia. J Anxiety Disord. 2006;20(7):961-972.

17. Keijsers GPJ, Kampman M, Hoogduin CA. Dropout prediction in cognitive behavior therapy for panic disorder. Behavior Therapy. 2001;32:739-749.

18. Hofmann SG, Barlow DH, Papp LA, et al. Pretreatment attrition in a comparative treatment outcome study on panic disorder. Am J Psychiatry. 1998;155(1):43-47. optimize clinical outcomes for existing disease states are major areas of interest. This journal has been accepted for indexing on PubMed Central. The manuscript management system is completely online and includes a very quick and fair peer-review system. Visit http://www.dovepress.com/ testimonials.php to read real quotes from published authors. 